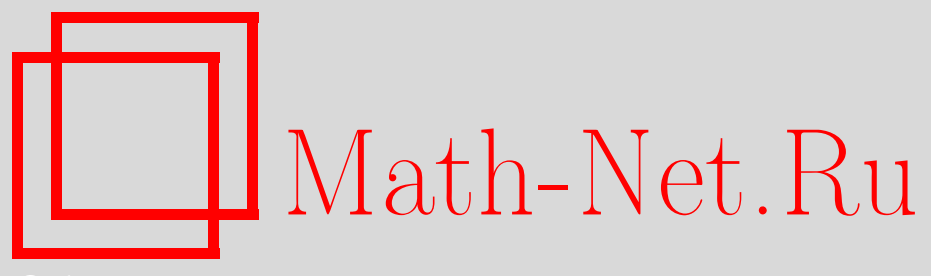

Д. А. Воротников, В. Г. Звягин, О траекторных и глобальных аттракторах для уравнений движения вязкоупругой среды, УМН, 2006, том 61, выпуск 2, 161-162

DOI: https://doi.org/10.4213/rm1695

Использование Общероссийского математического портала Math-Net.Ru подразумевает, что вы прочитали и согласны с пользовательским соглашением http://www . mathnet.ru/rus/agreement

Параметры загрузки:

IP : 34.229 .45 .116

26 апреля 2023 г., 13:03:24

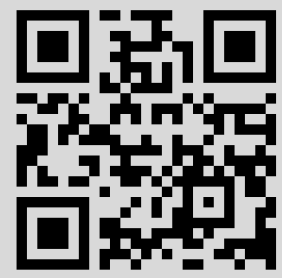




\title{
О траекторных и глобальных аттракторах для уравнений движения вязкоупругой среды
}

\author{
Д. А. Воротников, В. Г. Звягин
}

Пусть $E$ и $E_{0}$ - банаховы пространства, $E \subset E_{0}, E$ рефлексивно. Рассматривается абстрактное дифференциальное уравнение

$$
u^{\prime}(t)=A(u(t)), \quad u(t) \in E .
$$

В заметке изучаются аттракторы решений этого уравнения, принадлежащих пространству $C\left([0,+\infty) ; E_{0}\right) \cap L_{\infty}(0,+\infty ; E)$, с целью приложения к системам гидродинамики неньютоновских сред.

Пусть для уравнения (1) фиксировано некоторое семейство $\mathscr{H}^{+} \subset C\left([0,+\infty) ; E_{0}\right) \cap$ $L_{\infty}(0,+\infty ; E)$ его решений (сильных, слабых и т. п.), определенных на положительной полуоси $t \geqslant 0$. Множество $\mathscr{H}^{+}$будем называть пространством траекторий.

Определения. 1. Множество $P \subset C\left([0,+\infty) ; E_{0}\right) \cap L_{\infty}(0,+\infty ; E)$ называется притягивающим (для пространства траекторий $\mathscr{H}^{+}$), если для любого ограниченного в $L_{\infty}(0,+\infty ; E)$ множества $B \subset \mathscr{H}^{+}$и любого $M>0$ выполнено $\sup _{u \in B} \inf _{v \in P}\|T(h) u-v\|_{C\left([0, M] ; E_{0}\right)} \rightarrow 0$ при $h \rightarrow+\infty$. Здесь и ниже $T(h)$ обозначает оператор трансляции (сдвига): $T(h)(u)(t)=u(t+h) .2$. Притягивающее множество $P$ называется траекторным полуаттрактором, если $P$ компактно в $C\left([0,+\infty) ; E_{0}\right)$, ограничено в $L_{\infty}(0,+\infty ; E)$ и $T(t) P \subset P$ для всех $t \geqslant 0 . \quad 3$. Траекторный полуаттрактор $P$ называется траекторным аттрактором, если $T(t) P=P$ для всех $t \geqslant 0$. 4. Траекторный аттрактор называется минимальным, если он содержится в любом другом траекторном аттракторе. 5. Множество $\mathscr{A} \subset E$ называется глобальным аттрактором (в $\left.E_{0}\right)$ для пространства траекторий $\mathscr{H}^{+}$уравнения (1), если i) $\mathscr{A}$ компактно в $E_{0}$ и ограничено в $E$; ii) $\sup _{u \in B} \inf _{v \in \mathscr{A}}\|u(t)-v\|_{E_{0}} \rightarrow 0$ при $t \rightarrow \infty$ для любого ограниченного в $L_{\infty}(0,+\infty ; E)$ множества $B \subset \mathscr{H}^{+}$; iii) $\mathscr{A}$ содержится в любом множестве, удовлетворяющем условиям і) и ii). 6. Ядром $K(P)$ множества $P \subset C\left([0,+\infty) ; E_{0}\right) \cap L_{\infty}(0,+\infty ; E)$ называется множество $\left\{u \in L_{\infty}(-\infty,+\infty ; E) \mid \Pi_{+} T(t) u \in P \forall t \in \mathbb{R}\right\}$. Здесь $\Pi_{+}$обозначает оператор сужения на полуось $[0,+\infty)$.

Замечание. Если траекторный аттрактор для пространства траекторий $\mathscr{H}^{+}$содержится в $\mathscr{H}^{+}$, то он является минимальным. В [1] (при дополнительном ограничении $T(h) \mathscr{H}^{+} \subset \mathscr{H}^{+}$для всех $\left.h \geqslant 0\right)$ под траекторным аттрактором подразумевается траекторный аттрактор в смысле определения 3 , содержащийся в $\mathscr{H}^{+}$. Таким образом, используемое нами понятие минимального траекторного аттрактора является более широким, чем понятие траекторного аттрактора из [1], но обладает многими обычными свойствами траекторных аттракторов. В частности, минимальный траекторный аттрактор всегда порождает глобальный аттрактор (теорема 2). В то же время, этот более общий подход позволяет строить аттракторы без ограничений на пространство $\mathscr{H}^{+}$.

Теорема 1. Пусть существует траекторный полуаттрактор $P$ для $\mathscr{H}^{+}$. Тогда существует минимальный траекторный аттрактор $\mathscr{U} . \Pi$. При этом $\Pi_{+} K\left(\mathscr{H}^{+}\right) \subset$ $\mathscr{U}=\Pi_{+} K(\mathscr{U}) \subset \Pi_{+} K(P)$, а ядро $K\left(\mathscr{H}^{+}\right)$относительно компактно в $C((-\infty,+\infty)$; $\left.E_{0}\right)$ и ограничено в $L_{\infty}(-\infty,+\infty ; E)$.

Теорема 2. Если существует минимальный траекторный аттрактор $\mathscr{U}$ для $\mathscr{H}^{+}$, то существует глобальный аттрактор $\mathscr{A}$ для $\mathscr{H}^{+}$, причем для всех $t \geqslant 0: K\left(\mathscr{H}^{+}\right)(t) \subset \mathscr{A}=\mathscr{U}(t)=K(\mathscr{U})(t) . \quad$ Здесь $\mathscr{U}(t)=\{v(t) \mid v \in \mathscr{U}\}$, $K(P)(t)=\{v(t) \mid v \in K(P)\}$.

Работа выполнена при частичной поддержке РФФИ (грант № 04-01-00081). 
$\mathrm{C}$ помощью этих результатов строятся аттракторы краевой задачи для уравнений движения несжимаемой вязкоупругой среды с определяющим соотношением Джеффриса [2], описывающим растворы полимеров, битумы, бетоны, земную кору:

$$
\begin{gathered}
\frac{\partial u}{\partial t}+\sum_{i=1}^{n} u_{i} \frac{\partial u}{\partial x_{i}}-\mu_{1} \Delta u+\operatorname{grad} p=\operatorname{Div} \tau+f, \tau+\lambda_{1}\left(\frac{\partial \tau}{\partial t}+\sum_{i=1}^{n} u_{i} \frac{\partial \tau}{\partial x_{i}}\right)=2\left(\eta-\mu_{1}\right) \mathscr{E} \\
\operatorname{div} u=0,\left.\quad u\right|_{\partial \Omega}=0 .
\end{gathered}
$$

Здесь $\Omega$ - произвольная ограниченная область в $\mathbb{R}^{n}(n=2,3), u$ - неизвестный вектор скорости точек среды, $p$ - неизвестная функция давления, $\tau$ - неизвестный тензор (неньютоновская составляющая тензора касательных напряжений). Все они зависят от точки $x \in \Omega$ и момента времени $t . f(x)$ - плотность внешних сил, $\mathscr{E}=\mathscr{E}(u)=\left(\mathscr{E}_{i j}\right)$, $\mathscr{E}_{i j}=\frac{1}{2}\left(\frac{\partial u_{i}}{\partial x_{j}}+\frac{\partial u_{j}}{\partial x_{i}}\right)-$ тензор скоростей деформации, $\eta>0-$ вязкость среды, $\lambda_{1}>0-$ время релаксации, $\mu_{1}<\eta-$ положительная константа. Плотность среды считается единичной. Существование глобальных по времени сильных решений и единственность слабых решений для этой задачи являются открытыми проблемами. Также не удается построить класс существования слабых решений, для которого соответствующее пространство траекторий было бы одновременно инвариантно относительно оператора $T(t)$ и имело бы компактное ограниченное притягивающее множество.

Обозначения. $\mathbb{R}_{S}^{n \times n}$ - пространство симметричных матриц порядка $n \times n ; H, V$, $V_{\delta}(\delta \in(0,1])$ - замыкания множества $\mathscr{V}=\left\{u \in C_{0}^{\infty}\left(\Omega, \mathbb{R}^{n}\right), \operatorname{div} u=0\right\}$ соответственно в $L_{2}\left(\Omega, \mathbb{R}^{n}\right), W_{2}^{1}\left(\Omega, \mathbb{R}^{n}\right), W_{2}^{\delta}\left(\Omega, \mathbb{R}^{n}\right) ;\|\cdot\|$ - норма в $L_{2}\left(\Omega, \mathbb{R}^{n}\right)$ или $L_{2}\left(\Omega, \mathbb{R}^{n^{2}}\right)$ и $\|v\|_{1}=$ $\|\nabla v\|, v \in H^{1} ; C_{w}(J ; X)$ - пространство слабо непрерывных функций на промежутке $J \subset \mathbb{R}$ со значениями в некотором банаховом пространстве $X ; \gamma:=\min \left(\frac{1}{\lambda_{1}}, \frac{\mu_{1}}{2 K_{0}(\Omega)^{2}}\right)$, где $K_{0}(\Omega)$ - константа из неравенства Фридрихса $\|v\| \leqslant K_{0}(\Omega)\|v\|_{1}, v \in V$.

В качестве пространства $E$ берется $H \times L_{2}\left(\Omega, \mathbb{R}_{S}^{n \times n}\right)$, а в качестве $E_{0}$ - пространство $V_{\delta}^{*} \times H^{-\delta}\left(\Omega, \mathbb{R}_{S}^{n \times n}\right), \quad \delta \in(0,1] . \quad$ В качестве пространства траекторий $\mathscr{H}^{+}$для модели Джеффриса берется множество пар функций $(u, \tau)$, которые i) принадлежат классу $u \in L_{2, \text { loc }}(0,+\infty ; V) \cap L_{\infty}(0,+\infty ; H) \cap C_{w}([0,+\infty) ; H)$, $\tau \in L_{\infty}\left(0,+\infty ; L_{2}\right) \cap C_{w}\left([0,+\infty) ; L_{2}\right)$; ii) являются слабыми решениями задачи $(2)$, (точное определение см. в [3; задача (3.1), (3.2)]); iii) удовлетворяют энергетическому неравенству: $\frac{1}{2}\|u\|_{L_{\infty}(t, t+1 ; H)}^{2}+\frac{1}{4 \mu_{2}}\|\tau\|_{L_{\infty}\left(t, t+1 ; L_{2}\right)}^{2}+\frac{\mu_{1}}{2}\|u\|_{L_{2}(t, t+1 ; V)}^{2} \leqslant$ $e^{-2 \gamma t}\left(\|u\|_{L_{\infty}(0,+\infty ; H)}^{2}+\frac{1}{2 \mu_{2}}\|\tau\|_{L_{\infty}\left(0,+\infty ; L_{2}\right)}^{2}\right)+\frac{\gamma+1}{2 \mu_{1} \gamma}\|f\|_{V^{*} \text { при } t \geqslant 0 .}^{2}$

Теорема 3. Пусть $f \in V^{*}$. Для любых $a \in H, \tau_{0} \in L_{2}\left(\Omega, \mathbb{R}_{S}^{n \times n}\right)$ cуществует пара функиий (траектория) $(u, \tau) \in \mathscr{H}^{+}$, удовлетворяющая начальному условию $\left.u\right|_{t=0}=a,\left.\tau\right|_{t=0}=\tau_{0}$.

Теорема 4. Для модели Джеффриса существует минимальный траекторный аттрактор $\mathscr{U}_{J}$. В пространстве $H \times L_{2}$ существует глобальный аттрактор $\mathscr{A}_{J}$, т.е. минимальное компактное в $V_{\delta}^{*} \times H^{-\delta}$ и ограниченное в $H \times L_{2}$ притягивающее все траектории из $\mathscr{H}^{+}$множество. Для всех $t \geqslant 0 \quad K\left(\mathscr{H}^{+}\right)(t) \subset \mathscr{A}_{J}=\mathscr{U}_{J}(t)=$ $K\left(\mathscr{U}_{J}\right)(t)$.

\section{Список литературы}

[1] М. И. Вишик, В. В. Чепыжов, Матем. заметки, 71:2 (2002), 194-213. [2] М. Рейнер, Реология, Наука, M., 1965. [3] D. A. Vorotnikov, V. G. Zvyagin, Abstr. Appl. Anal., 2004, № 10, 815-829.

Д. А. Воротников (D. A. Vorotnikov)

Воронежский государственный университет E-mail: mitvorot@math.vsu.ru

В. Г. Звягин (V. G. Zvyagin)

Воронежский государственный университет

E-mail: zvyagin@alg.vsu.ru
Представлено В. М. Бухштабером Принято редколлегией 01.12 .2005 